\title{
A 10-year retrospective review of paediatric surgical oncology at Chris Hani Baragwanath Academic Hospital
}

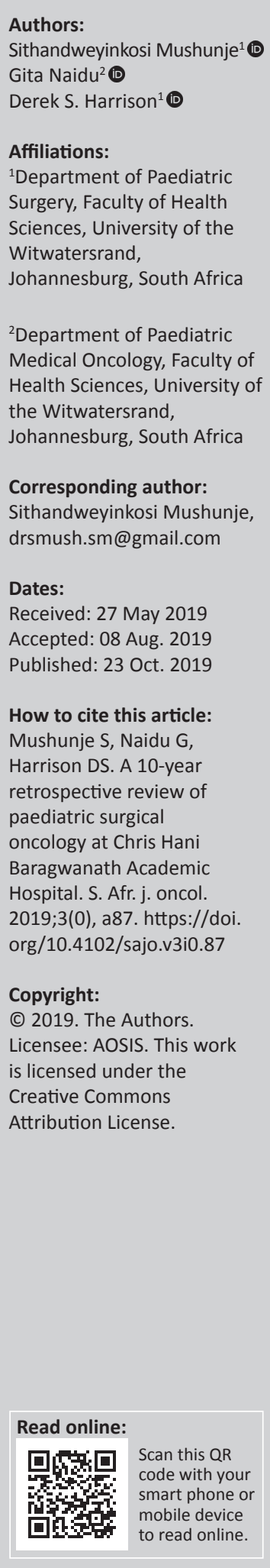

Background: There are few modifiable risk factors for solid tumours (STs) in children, except in the presence of a predisposing condition, and prevention is usually not possible; therefore, early diagnosis and prompt treatment are important in improving survival. Reporting the incidence, demographic data, presentation and outcomes of these STs improves awareness and allocation of resources for their management. There is a paucity of literature on the incidence, prevalence, demographics and treatment of paediatric STs in low- and middleincome countries (LMICs) including South Africa.

Aim: The aim of this study was to describe the demographics and spectrum of childhood STs at Chris Hani Baragwanath Academic Hospital (CHBAH).

Setting: The study was conducted at the Departments of Paediatric Surgery and Oncology at CHBAH.

Methods: A retrospective review of the records of children below 16 years of age with STs seen at $\mathrm{CHBAH}$ and managed by paediatric surgeons and oncologists from 01 January 2007 to 31 December 2016 was undertaken. The central nervous system, eye, bone and exclusively medically managed tumours were excluded.

Results: The solid tumours accounted for 33\% of all paediatric oncology cases. The three most common malignant tumours were: Wilms' tumour (32.7\%), rhabdomyosarcoma (18.2\%) and neuroblastoma (13.6\%). Solid tumours were most common in the 1 to 5 -year age group and most presented with advanced disease. An average of 30 oncological surgeries were performed per year.

Conclusion: The solid tumours studied represented a third of all oncology cases encountered in children; therefore, early diagnosis, early referral and prompt management of STs could improve childhood cancer survival.

Keyword: paediatric; solid tumours; oncology; improved survival; low- and middle-income countries.

\section{Introduction}

Childhood cancer is relatively uncommon, with the prevalence reported at $2 \%$ of all cancers in high income countries (HICs); however, after trauma, it is the second most common cause of death in HICs in children below 15 years of age. ${ }^{1,2,3}$ In low- and middle-income countries (LMICs), cancer deaths contribute a small percentage to childhood mortality; the leading causes of death remain diarrhoeal illnesses, pneumonia and other infectious diseases such as human immunodeficiency virus (HIV) and tuberculosis (TB). ${ }^{4,5}$ The incidence of childhood cancer in LMICs is quoted at $4 \%-15 \%$ of all cancers, which is significantly higher than that in HICs, probably owing to the greater proportion of children in LMICs. ${ }^{6,78,9,10}$

Paediatric surgical oncology is at the moment not regarded as a health care priority by policy makers in LMICs and sub-Saharan Africa (SSA). ${ }^{4}$ Although the fourth millennium development goal (MDG) was to reduce child mortality, ${ }_{1}^{11}$ most attention, research and resources were channelled towards the reduction of communicable diseases such as HIV and TB, which have been, for years, the leading causes of childhood mortality. ${ }^{5,12}$ With the reduction in these diseases, attention now also needs to be focused on non-communicable diseases (NCDs) including malignancies, which are emerging as a significant cause of childhood mortality in keeping with the World Cancer Declaration of the Union of International Cancer Control (UICC). ${ }^{12}$ The paucity of research and 
statistics in such NCDs makes it difficult to fully appreciate their burden and prioritise resources accordingly., ${ }^{4,12}$

There is a paucity of literature on childhood cancer in LMICs in general, $4,5,13$ and studies that have been published focused mainly on paediatric medical oncological cancers. The publication from the South African children's tumour registry (SACTR) reported on the incidence of different paediatric cancers, ${ }_{1}^{14}$ but no study in South Africa has focused on STs in detail. ${ }^{4,15,16,17}$

Generating incidence and prevalence data is important to understand the nature and magnitude of the problem for health care workers to maintain a high index of suspicion, prompting early diagnosis. ${ }^{5}$

The aim of this study therefore was to describe the demographics and spectrum of childhood STs at Chris Hani Baragwanath Academic Hospital (CHBAH), a hospital in SSA.

\section{Methods}

This was a retrospective descriptive study conducted at CHBAH's Departments of Paediatric Surgery and Paediatric Oncology. Files of children below 16 years of age with STs treated by paediatric surgeons and oncologists at $\mathrm{CHBAH}$ from 01 January 2007 to 31 December 2016 were reviewed.

An index case book kept at the Department of Paediatric Oncology was used to identify children with STs. Their files were then retrieved from the archives.

Children with Kaposi sarcoma, haemato-lymphoid tumours, brain, bone and eye tumours other than rhabdomyosarcoma and neuroblastoma were excluded from the study. Files of patients that were referred to $\mathrm{CHBAH}$ after surgery and received no further treatment from paediatric surgeons were also excluded from the data analysis.

A spreadsheet was formulated using Microsoft Excel. Each patient record was allocated a unique identification number to safeguard confidentiality. Data on the total number of all paediatric cancers seen each year were obtained from the Department of Paediatric Oncology for comparison.

The data in the spreadsheet were analysed using descriptive statistics. The relative frequencies of the different STs were calculated. Categorical data were expressed as frequencies and proportions. Continuous data were summarised using the mean and standard deviation (SD) if normally distributed, or median and interquartile range (IQR) if skewed. Pie charts, tables, histograms and bar graphs were used to simplify data interpretation.

\section{Ethical considerations}

No consent from individual patients was required as this was a retrospective study. Ethical clearance from the University of the Witwatersrand Human Research Ethics Committee
(Medical) was granted. The clearance number is M180152 and the certificate is attached.

\section{Results}

There were 387 children with STs included in the data analysis, accounting for $33 \%$ of all children with neoplasms seen over the 10-year period (Figure 1). The mean age at presentation was 5 years (SD 4.21), with a slight female predominance (M:F 1:1.3).

\section{Tumour distribution by age group}

The STs studied were most common in children between 1 and 5 years of age $(46.8 \%)$, followed by the $5-10$-year age group (19.6\%), the 10-16-year age group (17.1\%) and least common below 1 year of age (16.5\%) (Figure 2).

\section{Malignant tumours}

The 330 children with malignant STs were classified according to the 3rd edition of the International Classification of Childhood Cancer (ICCC-3) (Table 1). ${ }^{18}$

\section{Tumour distribution by histological subtype}

Malignant tumour proportions were also calculated according to the histological diagnosis regardless of site.

Nephroblastoma (Wilms' tumour [WT]) was the commonest histology, accounting for $32.7 \%$ of cases, followed by rhabdomyosarcoma (18.2\%), neuroblastoma (13.6\%), nonrhabdomyosarcomatous soft tissue sarcoma (STS) (12.1\%), carcinoma $(6.1 \%)$, malignant extracranial germ cell tumour (MEGCT) (5.2\%), hepatoblastoma (4.2\%) and peripheral primitive neuroectodermal tumour (pPNET) (2.4\%). Other malignant tumours not classified under the above histological diagnoses accounted for the remaining $5.5 \%$.

\section{Malignant renal tumours}

There were 124 children with malignant renal tumours, accounting for $37.6 \%(n=330)$ of the children with malignant STs.

\section{Nephroblastoma (Wilms' tumour)}

The 108 patients with WT, accounted for $32.7 \%$ of the children with malignant STs and $87.1 \%$ of those with malignant renal

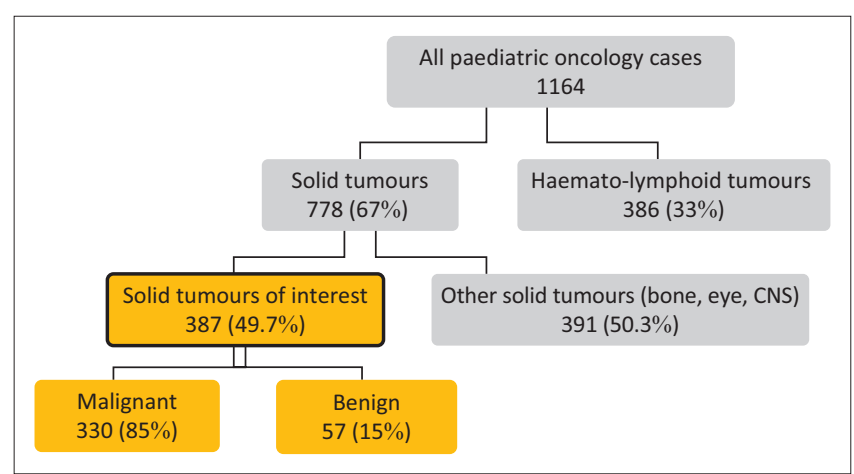

FIGURE 1: All tumours seen over a 10-year period. 


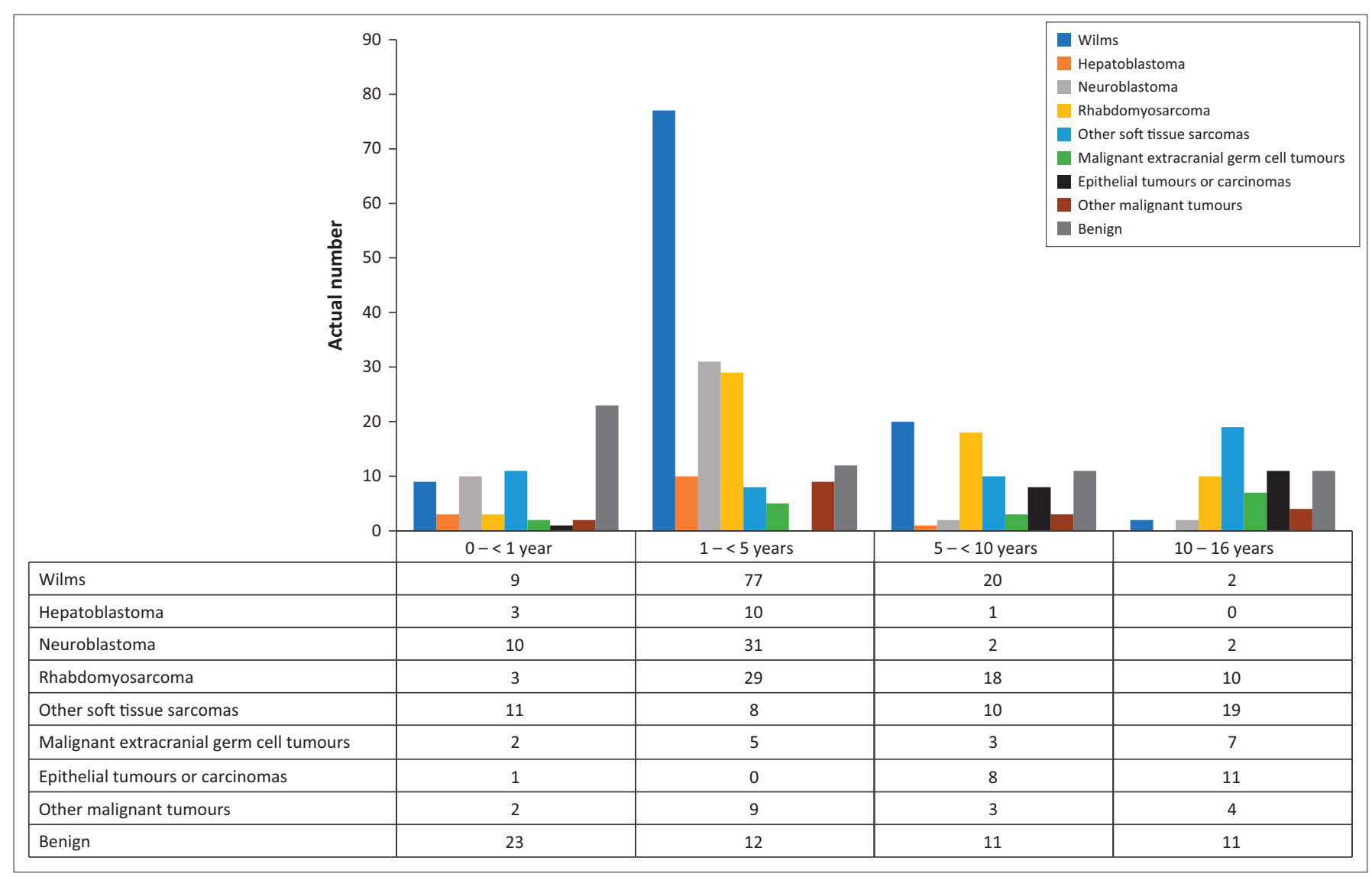

FIGURE 2: Age distribution of the tumours.

tumours. Seven children (6.5\%) had bilateral WT (stage 5). The male to female ratio was 1:1.3, with mean age at presentation of 3.68 years (SD 2.53).

The children with WT were staged according to the surgicopathological staging systems of the International Society of Paediatric Oncology (SIOP) and Children's Oncology Group (COG) ${ }^{19}$ A third (32.7\%) of the children with unilateral WT had distant metastases (stage 4) at presentation. The metastases were primarily to the lung $(74 \%)$, both the liver and lungs (16\%) and the liver alone (10\%). Another third $(32.7 \%)$ of the children had stage 1 disease, $16.8 \%$ had stage 2 disease and $17.8 \%$ had stage 3 disease.

Three of the children had known predisposing conditions, namely WAGR (WT, aniridia, genitourinary and mental retardation), Denys-Drash syndrome and hemihypertrophy.

\section{Other malignant renal tumours}

The second most frequent malignant renal tumour was the clear cell carcinoma of the kidney (CCSK) with nine cases $(7.3 \%)$, followed by the congenital mesoblastic nephroma in two infants (1.6\%), renal cell carcinoma (RCC) in two children $(1.6 \%)$, pPNET in another two cases $(1.6 \%)$ and an embryonal rhabdomyosarcoma in one child $(0.8 \%)$.

\section{Malignant primary liver tumours}

There were 24 children with malignant primary liver tumours, accounting for $7.3 \%$ of patients with malignant STs.

\section{Hepatoblastoma}

The 14 children with hepatoblastoma accounted for $4.2 \%$ of the children with malignant STs and $58 \%$ of malignant hepatic tumours. Their mean age at presentation was 2.61 years (SD 2.28) with an equal gender distribution. Children with hepatoblastoma were staged according to the COG PRE-Treatment EXTent of the tumour (PRETEXT). ${ }^{20}$ Fiftyseven per cent (8) had PRETEXT 4 tumours at presentation, 7\% (1) had PRETEXT 1 tumours, 29\% (4) PRETEXT 2 and the remaining $7 \%$ (1) had PRETEXT 3 tumours.

\section{Other malignant primary hepatic tumours}

Hepatocellular carcinoma (HCC) was seen in significantly older children, with a mean age of 10 years. Only one of the four children with HCC had chronic hepatitis B infection. There were five children with primary hepatic sarcomas; two had highgrade undifferentiated sarcomas, two had rhabdomyosarcomas (RMSs) (biliary) and one had a pPNET. One child presented with a ruptured primary neuroblastoma of the liver.

\section{Soft tissue sarcomas}

There were 108 children with STSs $(32.7 \%, n=330), 60(55.6 \%)$ had RMSs, 40 (37\%) had non-rhabdomyosarcomatous STSs (NRSTSs) and eight (7.4\%) had pPNETs.

\section{Rhabdomyosarcoma}

There were 60 children with RMS. Rhabdomyosarcoma was the second most common histological diagnosis accounting 
TABLE 1: Relative frequencies of malignant tumours according to the ICCC-3 classification.

\begin{tabular}{|c|c|c|c|}
\hline ICCC-3 group & Tissue of origin & Number & Percentage \\
\hline \multirow[t]{2}{*}{ IV } & $\begin{array}{l}\text { Neuroblastoma and other peripheral } \\
\text { nervous tissue tumours }\end{array}$ & 45 & 13.6 \\
\hline & Neuroblastoma & 45 & \\
\hline \multirow[t]{5}{*}{ VI } & Renal tumours & 121 & 36.7 \\
\hline & Nephroblastoma & 108 & \\
\hline & Clear cell sarcoma of the kidney & 9 & \\
\hline & Congenital mesoblastic nephroma & 2 & \\
\hline & Renal cell carcinoma & 2 & \\
\hline \multirow[t]{3}{*}{ VII } & Hepatic tumours & 18 & 5.5 \\
\hline & Hepatoblastoma & 14 & \\
\hline & Hepatocellular carcinoma & 4 & \\
\hline \multirow[t]{4}{*}{ IX } & Soft tissue and other extra-osseous tumours & 108 & 32.7 \\
\hline & Rhabdomyosarcoma & 60 & \\
\hline & Non-rhabdomyosarcoma soft tissue sarcoma & 40 & \\
\hline & $\begin{array}{l}\text { Peripheral primitive neuroectodermal } \\
\text { tumour (pPNET) }\end{array}$ & 8 & \\
\hline \multirow[t]{4}{*}{$x$} & $\begin{array}{l}\text { Germ cell tumours, trophoblastic tumours } \\
\text { and neoplasms of the gonad }\end{array}$ & 21 & 6.4 \\
\hline & Malignant gonadal germ cell tumour & 11 & \\
\hline & $\dagger$ Malignant extragonadal germ cell tumour & 6 & \\
\hline & Malignant sex cord stromal tumours & 4 & \\
\hline \multirow[t]{8}{*}{$\mathrm{XI}$} & $\begin{array}{l}\text { Other epithelial neoplasms and melanoma } \\
\text { Endocrine gland cancers }\end{array}$ & 14 & 4.2 \\
\hline & - Adrenocortical carcinoma & 2 & \\
\hline & - Papillary thyroid carcinoma & 2 & \\
\hline & Exocrine gland cancer & 1 & \\
\hline & - Pancreatic pseudopapillary carcinoma & 2 & \\
\hline & - Salivary muco-epidermoid carcinoma & 1 & \\
\hline & - Salivary acinic cell carcinoma & 4 & \\
\hline & $\begin{array}{l}\text { Melanoma } \\
\text { Squamous cell carcinoma }\end{array}$ & 2 & \\
\hline \multirow[t]{2}{*}{ XIIa } & Other specified malignant tumours & 3 & 0.9 \\
\hline & Malignant phaeochromocytoma & 3 & \\
\hline Total & - & 330 & 100.0 \\
\hline
\end{tabular}

$\dagger$. Excluding intracranial and intraspinal.

for $18.2 \%$ of patients with malignant STs. Sixty per cent (36) of the RMSs were embryonal, $31.7 \%$ (19) alveolar, and the remaining $8.3 \%$ (5) mixed. Children with RMS were commonly boys with a M:F ratio of 2.3:1. Their mean age at presentation was 6.25 (SD 4.19).

Twenty of the children with RMSs had head and neck tumours $(33.4 \%)$, and seven of these (35\%) were parameningeal. The second most common sites were the orbit and genitourinary system with nine cases each $(15 \%$ each). Eight children had paratesticular (13.3\%), six had extremity tumours $(10 \%)$ and eight had tumours from other rare sites $(13.3 \%)$. In $36(60 \%)$ of the children, the tumour occurred in favourable sites which included the orbit, head and neck other than parameningeal, paratesticular, biliary and genitourinary other than bladder/prostate. The 24 unfavourable sites $(40 \%)$ were parameningeal, bladder/ prostate, extremities and other rare sites.

RMSs were staged according to the COG pre-treatment tumour, node and metastasis (TNM) staging system. ${ }^{21,22}$ Fourteen of the children $(23.4 \%)$ had distant metastases at presentation. Twenty seven $(45 \%)$ had stage 1 disease, while $17(28.3 \%)$ and two (3.3\%) had stage 3 and 2 disease, respectively.

\section{Non-rhabdomyosarcoma soft tissue sarcomas}

These 40 cases accounted for $12.1 \%$ of the children with malignant STs. The children with NRSTSs showed two peaks in the age at presentation, in infancy and in adolescence. Their median age at presentation was 7 years (IQR 9.42), with a M:F ratio of 1:1. The NRSTSs included: five malignant peripheral nerve sheath tumours (MPNST) (12.5\%) and undifferentiated sarcomas (12.5\%); four fibrosarcomas (10\%), liposarcomas (10\%), synovial sarcomas (10\%), dermatofibrosarcoma protuberans $(10 \%)$ and desmoplastic small round cell tumours (DSRCT) $(10 \%)$; two alveolar soft part sarcomas (5\%), leiomyosarcomas $(5 \%)$ and extrarenal malignant rhabdoid tumours (5\%); a malignant triton tumour $(2.5 \%)$, an extraskeletal mesenchymal chondrosarcoma (2.5\%), an epithelioid haemangioendothelioma (2.5\%) and an angiosarcoma $(2.5 \%)$.

Children with NRSTSs were staged using the TNM system. ${ }^{22}$ Twelve (30\%) of the children had metastatic disease (stage 4) at presentation, another nine (22.5\%) presented with locally aggressive stage 3 disease precluding curative surgery. Fourteen (35\%) had stage 1 disease, and three $(7.5 \%)$ had stage 2 disease. Two patients (5\%) died of medical conditions before staging workup was complete. Four of the MPNST occurred in children with neurofibromatosis type 1 (NF1).

\section{Extraskeletal peripheral primitive neuroectodermal tumours}

There were eight children with extraskeletal pPNETs. They accounted for $2.4 \%$ of the children with malignant STs. Their median age at presentation was 11 years (IQR 10.83). These children were also staged using the TNM staging system. Two (25\%) patients had stage 1 disease, two (25\%) had stage 3 and the remaining four $(50 \%)$ had metastatic disease (stage 4 ).

\section{Peripheral neuroblastic tumours}

\section{Neuroblastoma}

Neuroblastoma was the third most common histological subtype. When classified according to the International Neuroblastoma Pathology Classification (the Shimada system) ${ }^{23}, 39$ children had neuroblastomas, six had ganglioneuroblastomas and four had ganglioneuromas. The patients with ganglioneuromas will not be discussed any further in this section as these tumours are benign. The 45 children with neuroblastomas accounted for $13.6 \%$ of the patients with malignant STs. Their mean age at presentation was 3.54 (SD 2.58). There was a slight female predominance with a $\mathrm{M}: \mathrm{F}$ ratio of $1: 1.3$.

Of the 45 patients with neuroblastoma, 55.5\% had adrenal primaries. The second most common site was the retroperitoneum $(17.8 \%)$, followed by the mediastinum $(8.9 \%)$, the pelvis $(2.2 \%)$ and the neck $(2.2 \%)$. The primary site was unknown in three patients $(6.7 \%)$, diagnosis being obtained from either a bone marrow trephine biopsy or fine needle aspiration (FNA) cytology of the subcutaneous metastases. Other uncommon sites $(6.7 \%)$ were the liver, the orbit and the head of the pancreas. The children with neuroblastoma were 
staged according to the International Neuroblastoma Risk Group (INRG) pre-operative staging system. ${ }^{23}$ Thirty-one of the 45 patients $(69 \%)$ presented with disseminated disease (stage M); only one of these had stage MS.

Two patients (4.4\%) had stage L1 and 12 patients (26.6\%) had L2 disease.

\section{Malignant extracranial germ cell tumours}

There were 17 children with MEGCTs, accounting for $5.2 \%$ of the patients with malignant STs. Eleven $(65 \%)$ of these children had gonadal (all ovarian), and six (35\%) had extragonadal tumours. Patients with gonadal MEGCTs were older children with a median age of 10 years at presentation (IQR 6.38). Seven of the 11 children (64\%) with gonadal MEGCTs had metastases at presentation, and two (18\%) had stage 3 disease. The remaining two children had stage 1 and 2 disease respectively ( $9 \%$ each).

Four of the children with extragonadal MEGCTs (66.6\%) had stage 1 disease, one had stage $2(16.7 \%)$ and the remaining one had stage 3 disease $(16.7 \%)$. These children presented at a mean age of 1.46 years (SD 1.8).

\section{Malignant sex cord stromal tumours}

There were four children with malignant sex cord stromal tumours (SCSTs) accounting for $1.2 \%$ of the patients with STs. All four tumours were ovarian, with only one child $(25 \%)$ presenting with distant metastasis (International Federation of Gynecology and Obstetrics [FIGO] stage 4). The remaining three children $(75 \%)$ had localised ovarian disease (FIGO stage 1). ${ }^{24}$

\section{Uncommon malignant tumours in the paediatric population}

\section{Epithelial tumours}

There were 20 children with carcinomas, accounting for $6.1 \%$ of the patients with malignant STs. These children presented at a mean age of 9.61 years (SD 3.73). Tumours included in this group were HCC, RCC, exocrine and endocrine carcinomas and skin cancers.

\section{Skin cancers}

Six children had skin cancers, presenting at a mean age of 10.2 years. Four had malignant melanoma and two had squamous cell carcinoma. Three of the four patients with malignant melanoma had a predisposing genetic condition, two had xeroderma pigmentosa and one had multiple congenital giant naevi. Fifty per cent (3) of the children with skin cancers had metastatic disease at presentation, and the remaining 50\% (3) had localised disease that was completely excised.

\section{Malignant endocrine tumours}

Seven patients with malignant endocrine tumours were encountered. Five of the children had adrenal endocrine tumours, three had malignant phaeochromocytoma and two had adrenocortical carcinoma. One child with an adrenocortical carcinoma had Li Fraumeni's syndrome. There were two children with papillary thyroid carcinomas. These endocrine tumours also occurred in older children and adolescents; the mean age was 10.1 years (SD 2.79).

\section{Malignant exocrine gland tumours}

Four children had malignant exocrine gland tumours. The three salivary gland tumours were the two mucoepidermoid carcinomas and an acinic cell carcinoma. One child had a pancreatic pseudopapillary carcinoma.

\section{Benign tumours}

There were 57 children with benign tumours (Table 2). Children with sacrococcygeal teratomas (SCTs) were the most common $(38.6 \%, n=57)$. Fifty-nine per cent of SCTs were diagnosed in the neonatal period, $82 \%$ within the first year of life and $100 \%$ by 5 years of age. One neonate with a SCT had Currarino syndrome.

\section{Oncological surgeries}

Three hundred and five oncological surgeries were performed, averaging 30 operations per year, 288 for the primary tumour and 17 for metastatic disease (Tables 3 and 4).

\begin{tabular}{|c|c|c|}
\hline Tissue of origin & Number & Percentage (\%) \\
\hline Germ cell tumours & 30 & 52.6 \\
\hline Gonadal & 5 & \\
\hline Extragonadal & - & \\
\hline - Sacrococcygeal teratoma & 22 & \\
\hline - †Other sites & 3 & \\
\hline Soft tissue tumours & 9 & 15.8 \\
\hline Neurofibroma & 4 & \\
\hline Tendon sheath fibroma & 1 & \\
\hline Dermatofibroma & 1 & \\
\hline Infantile fibrous hamartoma & 1 & \\
\hline Inflammatory myofibroblastic tumour & 1 & \\
\hline Nodular fasciitis & 1 & \\
\hline Other gonadal tumours & 5 & 8.8 \\
\hline Fibroma & 3 & \\
\hline Leydig cell tumour & 1 & \\
\hline Steroid cell tumour not otherwise specified & 1 & \\
\hline Endocrine and exocrine gland tumours & 5 & 8.8 \\
\hline Endocrine & 2 & \\
\hline -Paraganglioma & - & \\
\hline -Phaeochromocytoma & 1 & \\
\hline Exocrine & 1 & \\
\hline -Pleomorphic adenoma & - & \\
\hline Other & - & \\
\hline -Myoepithelioma of the breast & 1 & \\
\hline Neuroblastic & 4 & 7.0 \\
\hline Ganglioneuroma & 4 & \\
\hline Hepatic & 3 & 5.3 \\
\hline Haemangioendothelioma & 1 & \\
\hline Mesenchymal hamartoma & 2 & \\
\hline Renal & 1 & 1.7 \\
\hline Angiomyolipoma & 1 & \\
\hline Total & 57 & 100.0 \\
\hline
\end{tabular}

$\dagger$, Other sites were: anterior mediastinal, abdominal, thyroid 


\section{Palliative surgery}

Several palliative operations were carried out to improve the quality of life before eventual demise. These included four tracheostomies, a diverting colostomy, a vesicostomy and a gastrojejunostomy bypass.

\section{Discussion}

The relative frequencies of malignant STs expressed as a percentage of all paediatric cancers according to data from Europe, ${ }^{1}$ other South African data, ${ }^{5}$ and results from this

TABLE 3: Oncological surgeries for primary tumours.

\begin{tabular}{lcc}
\hline Type of operation & Number & Percentage \\
\hline Nephrectomy & 108 & 37.5 \\
Right & 58 & \\
Left & 50 & \\
Tumour excision & 106 & 37.0 \\
Primary excision & 94 & \\
Re-excision & 12 & \\
Adrenalectomy & 23 & 8.0 \\
Right & 10 & \\
Left & 13 & \\
Oophorectomy & 17 & 6.0 \\
Right & 8 & \\
Left & 9 & \\
Hemi-hepatectomy & 12 & 4.0 \\
Right & 11 & \\
Left & 1 & \\
†Orchidectomy & 10 & 3.5 \\
Laminectomy and spinal decompression & 3 & 1.0 \\
Parotidectomy & 3 & 1.0 \\
Hysterectomy & 1 & 0.7 \\
Total with BSO & 288.0 \\
Subtotal & 1 & \\
Total thyroidectomy & 2 & \\
Whipple's procedure & 1 & \\
Mastectomy & 2 & \\
\hline Total & 12 & \\
\hline
\end{tabular}

BSO, bilateral salpingo-oophorectomy.

$\dagger$, All but one orchidectomies for paratesticular rhabdomyosarcoma, one for a Leydig cell tumour. study are compared in Table 5. Whereas neuroblastoma is the commonest extracranial ST in $\mathrm{HICs}^{23}$ in South Africa, WTs and STSs are much more common than neuroblastoma. This trend was also shown in studies of cancer registries from different countries in SSA. ${ }^{5}$ This variation could be due to an interplay of environmental and genetic factors still to be investigated. The ethnic differences in children in HIC and SSA populations probably contribute to this discrepancy, as well as underdiagnosis of neuroblastoma in LMICs. ${ }^{5,25}$

Benign tumours represent more than $50 \%$ of all tumours in the paediatric population with a predominance of vascular tumours. ${ }^{2,26}$ In this study, the benign tumours were only $15 \%$; this can be explained by the fact that not all benign tumours may have been referred to the oncology department which was the data repository for this review. A more representative study for benign tumours would have to include other benign STs managed by surgeons alone by collecting data from both departments.

Wilms' tumour was the commonest extracranial solid tumour. Whereas patients were assigned a stage, it was unclear from the files which protocol (SIOP vs. COG) the oncology unit followed during the study period; this may lead to inaccuracy

TABLE 5: Comparison of relative tumour frequencies in the different regions.

\begin{tabular}{lccc}
\hline Tumour & International $\uparrow(\%)$ & RSA: (\%) & CHBAH (\%) \\
\hline Neuroblastoma & 7.6 & 5.7 & 3.9 \\
Wilms & 5.6 & 13.5 & 9.3 \\
RMS & 3.4 & 5.2 & 5.2 \\
GCT $\S$ & 2.2 & 3.8 & 1.4 \\
Hepatoblastoma & 1.0 & 1.5 & 1.2 \\
Carcinomas & 1.1 & 3.8 & 2.1 \\
\hline
\end{tabular}

CHBAH, Chris Hani Baragwanath Academic Hospital; RSA, Republic of South Africa; GCT, germ cell tumour; RMS, rhabdomyosarcomas.

$\%$ depicted here for all studies are proportions of all childhood cancers.

$\dagger$, Data from Europe. ${ }^{1}$

$\$$ Data from RSA in 2008-2012. ${ }^{5}$

$\S$, GCT exclude intracranial and intraspinal in all studies.

TABLE 4: Operations for metastatic disease.

\begin{tabular}{|c|c|c|}
\hline Type of operation & Primary tumour & Histology of suspected metastasis \\
\hline Right hepatectomy & WT & WT \\
\hline Left hepatectomy & WT & WT \\
\hline Right thoracotomy & Undifferentiated sarcoma & No masses found \\
\hline Right thoracotomy and excision of nodules & WT & WT \\
\hline Left thoracotomy and lower lobe segmentectomy & WT & WT \\
\hline Left thoracotomy and excisional biopsy & WT & WT \\
\hline Right thoracotomy $\dagger$ & Malignant triton tumour & Malignant triton tumour with involved margins \\
\hline Right thoracotomy and right lower lobectomy $\dagger$ & Malignant triton tumour & Malignant triton tumour \\
\hline Right thoracotomy and biopsy & WT & Tuberculosis \\
\hline Thoracoscopic lung biopsies & WT & WT \\
\hline Thoracoscopic lung biopsies & WT & WT \\
\hline Thoracoscopic lung biopsies & WT & No tumour found \\
\hline Sternotomy and bilateral metastesectomy & WT & Fibrous tissue, no neoplasm \\
\hline Sternotomy and bilateral metastesectomy & WT & Fibrosis, no neoplasm \\
\hline Sternotomy and bilateral metastesectomy & Osteosarcoma & Osteosarcoma \\
\hline Sternotomy and bilateral metastesectomy & Osteosarcoma & Osteosarcoma \\
\hline
\end{tabular}

WT, Wilms' tumour.

$\dagger$, Same patient. 
in data analysis and interpretation. A unit protocol that follows mainly the SIOP UMBRELLA protocol, ${ }^{27}$ has since been formulated that has standardised care of patients with WT.

Although there were discrepancies in the frequencies of different tumours when compared to HICs, the age at presentation and gender distribution of most of the tumour subtypes were in keeping with existing literature..$^{22,28,29}$

Fifty per cent of gonadal tumours in this review were malignant, which is much higher than the expected $10 \%{ }^{30}$ This seemingly higher proportion of malignant gonadal tumours may be because some benign gonadal tumours were not referred to oncology and hence not captured.

Predisposing conditions were found in 13 cases (3\%). Five of these 13 children who developed malignant tumours were already on surveillance and had stage 1 disease at diagnosis. If, therefore, only $3 \%$ of the tumours could have been diagnosed early through surveillance, there is need for health care workers and caregivers to maintain a high index of suspicion for early diagnosis of the remaining $97 \%$ of the tumours.

\section{Conclusion}

This study is a clinical audit of the spectrum of STs managed by paediatric surgeons and paediatric oncologists in a single centre. Incomplete data and missing records were the main problems faced during data collection. Twenty patients were excluded based on missing records, which may have affected the results and interpretation. Whereas the study carries the limitations of a single-centre retrospective review, it has exposed areas that can be improved, both at $\mathrm{CHBAH}$ and in South Africa in general. The importance of good record keeping should be emphasised.

Children with STs accounted for a third of all oncology cases encountered in children at $\mathrm{CHBAH}$, with a significant proportion of the malignant tumours presenting late with advanced disease. Whereas caregivers are often held responsible for this late presentation, no programmes have been put in place to create awareness on signs and symptoms of paediatric STs, and certainly some health care workers have also missed signs which contributed to delayed referral.

Awareness campaigns on the warning and danger signs of childhood cancer that involve the community, with flyers at local clinics in local languages, and outreach programmes, may encourage early presentation and early referral, that will hopefully improve survival in paediatric STs at CHBAH and in South Africa.

A surgeon is an important member of the multi-disciplinary team managing paediatric surgical oncology.

Most units in South Africa have few or no paediatric oncological surgeons. Training programmes for paediatric surgeons with interest in oncology may be valuable.

\section{Acknowledgements}

The authors acknowledge the Department of Paediatric Surgery Research Unit and the Department of Paediatric Oncology at CHBAH for the support they offered during the period spent in collecting data. The authors extend special thanks to Dikopo Fihla and Charles Mbatha for their help in locating files from archives.

\section{Competing interests}

The authors declare that they have no financial or personal relationships that may have inappropriately influenced them in writing this article.

\section{Authors' contributions}

S.M. (principal investigator) wrote up the research proposal, collected and analysed the data and wrote this manuscript with the help of the supervisors. G.N. (second supervisor) provided direction and leadership from the writing of the protocol through data collection to the writeup and made corrections at each stage which were incorporated into this article. D.S.H. (main supervisor) provided direction and leadership from the writing of the protocol through data collection to the write-up and made corrections at each stage which were incorporated into this article.

\section{Funding}

No funding was obtained from any source. The expected cost of publishing will be covered by the principal investigator with the help of the University of the Witwatersrand Department of Paediatric Surgery Research Unit.

\section{Data availability statement}

Data sharing is not applicable to this article as no new data were created or analysed in this study.

\section{Disclaimer}

The views and opinions expressed in this article are those of the authors and do not necessarily reflect the official policy or position of any affiliated agency of the authors.

\section{References}

1. Kaatsch P. Epidemiology of childhood cancer. Cancer Treat Rev. 2010;36(4): 277-285. https://doi.org/10.1016/j.ctrv.2010.02.003

2. Sharma S, Mishra K, Agarwal S, Khanna G. Solid tumors of childhood. Indian J Pediatr. 2004;71(6):501-504. https://doi.org/10.1007/BF02724289

3. Rodwin RL, Kupfer GM. Childhood cancer epidemiology. Medscape. [cited 2018 Aug 18]. Available from: https://emedicine.medscape.com/article/989841overview\#a2

4. Hadley LGP, Rouma BS, Saad-Eldin Y. Challenge of pediatric oncology in Africa. Semin Pediatr Surg. 2012;21(2):136-141. https://doi.org/10.1053/j.sempedsurg. 2012.01.006

5. Stefan C, Bray F, Ferlay J, Liu B, Parkin DM. Cancer of childhood in sub-Saharan Africa. Ecancermedicalscience. 2017;11:1-95. https://doi.org/10.3332/ecancer. 2017.755

6. Shah $\mathrm{SH}$, Pervez $\mathrm{S}$, Hassan SH. Frequency of malignant solid tumors in children. J Pak Med Assoc. 2000;50(3):86-88. 
7. Magrath I, Steliarova-Foucher E, Epelman S, et al. Improving cancer care for children and young people 2 Paediatric cancer in low-income and middle-income 2045(13)70008-1

8. Israels T, Ribeiro RC, Molyneux EM. Strategies to improve care for children with cancer in sub-Saharan Africa. Eur J Cancer. 2010;46(11):1960-1966. https://doi. org/10.1016/j.ejca.2010.03.027

9. Martin VA. Mid-year population estimates 2017 [homepage on the Internet] Stats Sa, 2017 [cited 2018 Sep 19]; p. 7-8. Available from: www.statssa.gov.za.

10. Butler EK, Tran TM, Nagarajan N, et al. Epidemiology of pediatric surgical needs in low-income countries. PLoS One. 2017;12(3):1-9. https://doi.org/10.1371/journal. pone.0170968

11. United Nations. Millennium development goals [homepage on the Internet]. 2015 [cited 2019 Jul 07]. Available from: http://www.un.org/millenniumgoals/2015 MDG_Report/pdf/MDG 2015 rev (July 1).pdf

12. Rodriguez-Galindo C, Friedrich P, Morrissey L, Frazier L. Global challenges in pediatric oncology. Curr Opin Pediatr. 2013;25(1):3-15. https://doi.org/10.1097/ MOP.0b013e32835c1cbe

13. Kruger M, Hendricks M, Davidson A, et al. Childhood cancer in Africa. Pediat Blood Cancer. 2014;61:587-592. https://doi.org/10.1002/pbc.24845

14. Stefan DC, Stones DK, Wainwright D, et al. Childhood cancer incidence in South Africa, 1987-2007. S Afr Med J. 2015;105(11):939. https://doi.org/10.7196/ SAMJ.2015.v105i11.9780

15. Hesseling PB, Wessels G, Van Riet FA. The Tygerberg Hospital children's tumou registry 1983-1993. Eur J Cancer. 1995;31(9):1471-1475. https://doi.org/10.1016/ 0959-8049(95)00289-U

16. Stones DK, De Bruin GP, Esterhuizen TM, Stefan DC. Childhood cancer survival rates in two South African units. S Afr Med J. 2014;104(7):501-504. https://doi. org/10.7196/SAMJ.7882

17. Erdmann F, Kielkowski D, Schonfeld SJ, et al. Childhood cancer incidence patterns by race, sex and age for 2000-2006: A report from the South African National Cancer Registry. Int J Cancer. 2015;136(11):2628-2639. https://doi.org/10.1002/ ijc.29308
18. Steliarova-Foucher E, Stiller C, Lacour B, Kaatsch P. International classification of childhood cancer, third edition. Cancer. 2005;103(7):1457-1467. https://doi.org/ $10.1002 /$ ijc. 29308

19. Irtan S, Ehrlich PF, Pritchard-Jones K. Wilms tumor: "State-of-the-art" update, 2016. Semin Pediatr Surg. 2016;25(5):250-256. https://doi.org/10.1053/j. sempedsurg.2016.09.003

20. Stringer MD. Liver tumors. Semin Pediatr Surg. 2000;9(4):196-208. https://doi. org/10.1053/spsu.2000.18844

21. Levy SM, Hetz RA, Letourneau PA, Andrassy RJ. Chapter 70 - Rhabdomyosarcoma. In: Holcomb GW III, Murphy PJ, Ostlie DJ, editors. Ashcraft's pediatric surgery. 6th ed. Philadelphia, PA: Elsevier, 2014; p. 979-988.

22. Agarwala S. Pediatric rhabdomyosarcomas and nonrhabdomyosarcoma soft tissue sarcoma. J Indian Assoc Pediatr Surg. 2006;11(1):15

23. Davidoff AM. Neuroblastoma. Semin Pediatr Surg. 2012;21(1):2-14.

24. Prat J. FIGO staging classification for cancer of the ovary, fallopian tube, and peritoneum: Abridged republication. J Gynaecol Oncol 2014;26(2):87-89.

25. Carter N, Avery A, Libes J, Lovvorn H, Hansen E. Pediatric solid tumors in resourceconstrained settings: A review of available evidence on management, outcomes, and barriers to care. Children. 2018;5(11):143.

26. Kundu R, Jindal G, Dalal U, Mohan H, Punia R, Mundi I. Spectrum of nonhematological pediatric tumors: A clinicopathologic study of 385 cases. Indian J Med Paediatr Oncol. 2014;35(2):170.

27. Van Den Heuvel-Eibrink MM, Hol JA, Pritchard-Jones K, et al. Position paper: Rationale for the treatment of Wilms tumour in the UMBRELLA SIOP-RTSG 2016 protocol. Nat Rev Urol. 2017;14(12):743-752.

28. Kline NE, Sevier N. Solid tumours in children. J Pediatr Nurs. 2003;18(2): 96-102.

29. Ehrlich PF, Shamberger RC. Chapter 65 - Renal tumors. In: Holcomb GW III, Murphy PJ, Ostlie DJ, editors. Ashcraft's pediatric surgery. 6th ed. Philadelphia: Elsevier 2014; p. 859-882.

30. Strickland JL. Pediatric and adolescent gynecology. In: Ashcraft's pediatric surgery. In: Holcomb GW III, Murphy PJ, Ostlie DJ, editors. 6th ed. Philadelphia, PA: Elsevier, 2014; p. 1046-1057. 